\title{
Release timescales of solar energetic particles in the low corona
}

\author{
N. Agueda ${ }^{1}$, K.-L. Klein ${ }^{2}$, N. Vilmer ${ }^{2}$, R. Rodríguez-Gasén ${ }^{2}$, O. E. Malandraki ${ }^{3}$, A. Papaioannou ${ }^{3}$, \\ M. Subirà ${ }^{1}$, B. Sanahuja ${ }^{1}$, E. Valtonen ${ }^{4}$, W. Dröge ${ }^{5}$, A. Nindos ${ }^{6}$, B. Heber ${ }^{7}$, \\ S. Braune ${ }^{8}$, I. G. Usoskin ${ }^{9}$, D. Heynderickx ${ }^{10}$, E. Talew ${ }^{11}$, and R. Vainio ${ }^{4,11}$ \\ 1 Departament d'Astronomia i Meteorologia, Institut de Ciències del Cosmos, Universitat de Barcelona, 08028 Barcelona, Spain \\ e-mail: n.agueda@ub.edu \\ 2 LESIA-UMR 8109, Observatoire de Paris, CNRS, Univ. Paris 6 \& 7, 92195 Meudon, France \\ 3 IAASARS, National Observatory of Athens, 5236 Penteli, Greece \\ 4 Department of Physics and Astronomy, University of Turku, 20014 Turku, Finland \\ 5 Lehrstuhl für Astronomie, Julius-Maximilians Universität Würzburg, 97074 Würzburg, Germany \\ 6 Department of Physics, Section of Astrogeophysics, University of Ioannina, 45110 Ioannina, Greece \\ 7 Institut für Experimentelle und Angewandte Physik, Christian-Albrechts-Universität zu Kiel, 24118 Kiel, Germany \\ 8 Solar Physics Group, Leibniz Institut für Astrophysik Potsdam, 14482 Potsdam, Germany \\ 9 Sodankylä Geophysical Observatory, Oulu Unit, University of Oulu, 90014 Oulu, Finland \\ 10 DH Consultancy BVBA, 3000 Leuven, Belgium \\ 11 Department of Physics, University of Helsinki, 00014 Helsinki, Finland
}

Received 31 January 2014 / Accepted 5 August 2014

\section{ABSTRACT}

\begin{abstract}
Aims. We present a systematic study of the timing and duration of the release processes of near-relativistic $(\mathrm{NR} ;>50 \mathrm{keV})$ electrons in the low corona.

Methods. We analyze seven well-observed events using in situ measurements by both the ACE and Wind spacecraft and context electromagnetic observations in soft X-rays, radio, hard X-rays and white light. We make use of velocity dispersion analysis to estimate the release time of the first arriving electrons and compare with the results obtained by using a simulation-based approach, taking interplanetary transport effects into account to unfold the NR electron release time history from in situ measurements.

Results. The NR electrons observed in interplanetary space appear to be released during either short $(<30 \mathrm{~min})$ or long ( $>2 \mathrm{~h}$ ) periods. The observation of NR electron events showing beamed pitch-angle distributions (PADs) during several hours is the clearest observational signature of sustained release in the corona. On the other hand, the in situ observation of PADs isotropizing in less than a couple of hours is a clear signature of a prompt release of electrons in the low corona. Short release episodes appear to originate in solar flares, in coincidence with the timing of the observed type III radio bursts. Magnetic connectivity plays an important role. Only type III radio bursts reaching the local plasma line measured at $1 \mathrm{AU}$ are found to be related with an associated release episode in the low corona. Other type III bursts may also have a release of NR electrons associated with them, but these electrons do not reach L1. Long release episodes appear associated with signatures of long acceleration processes in the low corona (long decay of the soft $\mathrm{X}$-ray emission, type IV radio bursts, and time-extended microwave emission). Type II radio bursts are reported for most of the events and do not provide a clear discrimination between short and long release timescales.
\end{abstract}

Key words. Sun: particle emission - Sun: flares - Sun: radio radiation - Sun: coronal mass ejections (CMEs) - interplanetary medium

\section{Introduction}

Solar energetic particle (SEP) events are observed by spacecraft in the heliosphere in association with solar transient eruptive events, such as flares and coronal mass ejections (CMEs). Synergies between radio diagnostics of flare and CMEassociated phenomena and information from optical, UV, soft $\mathrm{X}$-ray (SXR) and hard X-ray (HXR) data provide unprecedented opportunities for the study of these phenomena and their relation to SEP particle release processes (e.g. Rouillard et al. 2012). The radio emission has a rich diagnostic potential on the location and temporal evolution of the particle release processes from the corona into the interplanetary medium, and it often reveals a complex, multi-impulsive or quasi-continuous structure (Maia $\&$ Pick 2004; Klein et al. 2005).

in situ measurements of SEPs refer to a sample of the particles released into the heliosphere. In some cases, this can be an incomplete sample of the electrons accelerated during a solar event, as not all of them may have access to interplanetary space (Krucker et al. 2007; Klein et al. 2010), and those which do have access may be injected into several coronal structures (Klein et al. 2005). Open magnetic flux tubes expand rapidly and cover several tens of degrees in longitude on the source surface, and some of these open field lines are found to connect the parent active region to the footpoint of the nominal Parker spiral, even when the parent active region is as far as $50^{\circ}$ away (Klein et al. 2008). The SEPs measured in situ are the sample of particles released onto the flux tube connecting the spacecraft with the Sun.

Observations of the same SEP event from different locations in the heliosphere give important clues for understanding coronal acceleration processes and interplanetary SEP propagation. A common result from the Helios and Ulysses missions was that the biggest events seen at Earth were also seen in the inner/outer 
heliosphere even when the spacecraft location was very remote from the longitude/latitude of the solar event (Wibberenz \& Cane 2006; Lario et al. 2003). STEREO multi-spacecraft observations confirmed the large spread of SEP events (e.g. Dresing et al. 2012; Wiedenbeck et al. 2013).

In most observational studies, the release time of the first arriving particles is inferred from either the dispersion in velocity observed during the event onset time (e.g. Krucker et al. 1999) or by assuming that the first arriving electrons propagate scatter-free along an Archimedean interplanetary magnetic field line (Haggerty \& Roelof 2002). Krucker et al. (1999) and Haggerty \& Roelof (2002) found that in few cases the inferred release times were consistent with the timing of the type III radio emission, but the electrons were apparently released up to half an hour later than the type III burst in many cases. For the origin of these delays, four explanations were proposed: (i) delayed electrons come from coronal shocks (Krucker et al. 1999; Simnett et al. 2002); (ii) all electrons originate from a single population, and the delays are due to interplanetary transport effects (Cane 2003); (iii) the CME shock front plays a minor role and the delayed electrons originate at a reconnection site behind it (Maia \& Pick 2004; Klein et al. 2005); and (iv) the delays are apparent, produced by pre-event background and cross-talk between energy channels, as well as interplanetary transport effects (Laitinen et al. 2010; Haggerty \& Roelof 2003; Lintunen \& Vainio 2004; Sáiz et al. 2005). In any case, a timing comparison between the apparent release time of the earliest electrons and the electromagnetic (EM) signatures of an event has proved to be inconclusive in many cases (Klein et al. 2005).

An actual modeling of the SEP transport in the heliosphere is fundamental for removing all the uncertainties inherent in the analysis of in situ SEP observations. To unfold the SEP release time history from in situ measurements, it is necessary to deconvolve the effects of interplanetary particle transport from the acceleration site to the spacecraft by using the results of an interplanetary transport model (e.g. Ruffolo et al. 1998; Maia et al. 2007; Agueda et al. 2008). To properly model an SEP event, it is imperative to make use of directional intensities (or anisotropy measurements) to constrain the problem (Agueda et al. 2009b).

We present a systematic study of the timing and duration of the release processes of near-relativistic electrons (NR; $>50 \mathrm{keV}$ ) in the low corona by taking interplanetary transport effects into account. We use in situ measurements by both ACE and Wind and context EM observations in SXR, radio, HXR and white light (Sect. 2). We review the data-driven and simulationbased analysis methods used to infer the release time of the first arriving electrons and the release-time history near the Sun (Sect. 3). We select a sample of well-observed events (Sect. 4) suitable for simulation-based analyzes using the database of results of an interplanetary transport model (Agueda et al. 2012). Modeling results are presented and discussed in Sects. 5 and 6. Finally, Sect. 7 gives the conclusions of this work.

\section{Observations}

We use in situ measurements of energetic electrons by the Electron, Proton and Alpha Monitor (EPAM) experiment on board the Advanced Composition Explorer (ACE) spacecraft (Gold et al. 1998). The EPAM measures NR electrons in the energy range between 40 and $300 \mathrm{keV}(0.4-0.7 c)$. Observations are extended using energetic electron data from the ThreeDimensional Plasma Analyzer (3DP) experiment on the Wind spacecraft (Lin et al. 1995) in similar energy ranges.
Both sets of observations include directional distributions of NR electrons. Detectors on board spin-stabilized spacecraft, such as ACE and Wind, use the rotation of the spacecraft to scan different regions of space with a single detector. The swath of space swept out by a detector during a spin is divided into nearly equally spaced sectors. By measuring the magnetic field vector in the spacecraft coordinate system, it is possible to infer the pitch-angle of the particles being measured by each sector. We use measurements of the LEFS60 telescope on board ACE/EPAM, which points $60^{\circ}$ away from the spin axis and allows measurements in eight different sectors (Gold et al. 1998). The Wind/3DP experiment uses several rotating detectors, oriented at different degrees from the spin axis, to scan the full $4 \pi$, which allows the calculation of the complete pitch-angle distributions.

We consider context EM observations of the parent solar activity. Associations are made primarily on the basis of location and timing information. We use observations reported by the Solar Geophysical Data (US Department of Commerce, Boulder, CO, USA) of the location of the associated $\mathrm{H} \alpha$ activity. In addition, we include the time history of the whole Sun SXR flux observed by the GOES satellite, HXR observations from the Reuven Ramaty High-Energy Solar Spectroscopic Imager (RHESSI; Lin et al. 2002), whenever available, and white-light observations of CMEs reported in the SOHO/LASCO CME catalog (Yashiro et al. 2004).

Decametric-to-kilometric radio spectra by the WAVES experiment (Bougeret et al. 1995) on board the Wind spacecraft are included. The start and end times of the type III emission are estimated near $4 \mathrm{MHz}$ requiring the radio flux to be at least 3 standard deviations above the background. Type II timings are listed as reported in the Wind/WAVES website ${ }^{1}$. Additional data from ground-based observatories at metric wavelengths are discussed for those events that occurred during the European day-time; i.e., with observations from ground-based observatories available in SEPServer $^{2}$ (Vainio et al. 2013).

\section{Data-driven versus simulation-based analysis methods}

Solar energetic particles are observed in the heliosphere after they have propagated a long distance in the interplanetary medium, where they were scattered off the fluctuations in the interplanetary magnetic field (IMF). This can smear out the temporal evolution of the solar release processes.

\subsection{Direct data analysis methods}

Direct data analysis methods are frequently employed to estimate the solar release time of the first arriving particles at $1 \mathrm{AU}$. The solar release time, $t_{\odot}$, can be estimated by shifting back in time the onset time of the electron event at $1 \mathrm{AU}, t_{\oplus}$, by the time the electrons need to travel from the Sun to $1 \mathrm{AU}, \Delta t$; that is,

$t_{\odot}(E)=t_{\oplus}(E)-\Delta t=t_{\oplus}(E)-\frac{L}{v(E)}$,

where $L$ is the apparent path length and $v$ is the speed of a particle with energy $E$.

One can use the average solar wind speed value measured at the spacecraft location to estimate the length of the IMF line connecting the source to the observer, $L$, under the approximation

\footnotetext{
http://www-lep.gsfc.nasa.gov/waves/

2 http://server.sepserver.eu
} 
that the IMF can be described by a Parker spiral. Then, under the assumption of interplanetary scatter-free transport, the solar release time can be calculated by determining the event onset time in one energy channel and shifting it in time according to the time of flight of the electrons (e.g. Haggerty \& Roelof 2002). This data analysis method is usually referred to as time-shifting analysis.

Assuming simultaneous release of particles at all energies and subsequent propagation through an energy-independent path length before detection, the observed onset times can be used in a velocity dispersion analysis (VDA). Then the release time and the length of the interplanetary trajectory are inferred from a linear fit to the observed onset times as a function of each channel inverse speed (see e.g. Krucker et al. 1999).

Both data analysis methods rely on the event onset time determination. The simplest approach to estimate the event onset time consists in calculating the time when the intensities reach a threshold above the pre-event background intensities, given by $I_{b}+n \sigma$, where $I_{b}$ is the mean pre-event background intensity, $\sigma$ is the standard deviation, and $n$ is an integer (e.g. Krucker et al. 1999; Malandraki et al. 2012). The event onset time can also be determined using more sophisticated methods such as the Poisson-CUSUM method (Huttunen-Heikinmaa et al. 2005).

For those events with small signal-to-noise ratios, we note that the first arriving particles are more likely masked by the pre-event background. The event onset time may also appear delayed when the event exhibits a ramp profile, which is slowly rising above background. Therefore, the most accurate onset time determination is obtained for large SEP events with a prompt rising phase and no significant background from previous events. Another effect that might blur the event onset time determination is related to particle contamination by high energy electrons producing a response in the low energy channels during the rising phase of an SEP event (Haggerty \& Roelof 2003; Tan et al. 2011).

Besides the inherent uncertainties in the onset time determination, numerical simulations have shown that the VDA can produce misleading results, providing incorrect release times and path lengths that deviate greatly from the actual path length, when interplanetary scattering effects are important (Lintunen \& Vainio 2004; Sáiz et al. 2005). Furthermore, Laitinen et al. (2010) showed that differences in the pre-event background and the event peak spectra can greatly affect the accuracy of the determination of the solar release time using a VDA, as the preevent background may mask the event onset time to a different extent in different energy channels. The critical assumptions in the VDA and some conflicting results from its use were reviewed by Kahler \& Ragot (2006).

\subsection{Inversion methodology}

Numerical simulations of the propagation of SEPs along the IMF are a useful tool to understand the sources of SEP events. We currently have a good theoretical understanding of the transport processes that affect SEPs in the interplanetary medium (Jokipii 1966; Roelof 1969; Ruffolo 1995; Dröge 2003; Dalla et al. 2013), and it is possible to model the processes undergone by energetic particles during their propagation from the source to the observer (e.g. Ruffolo 1995; Kocharov et al. 1998; Dröge et al. 2010; Kelly et al. 2012; Marsh et al. 2013).

In the absence of large-scale disturbances, the IMF can be described as an average field, given by an Archimedean spiral, with a superposed turbulent component. The propagation of charged particles along the IMF has then two components, adiabatic motion along the smooth field and pitch-angle scattering by magnetic turbulence. The quantitative treatment of the evolution of the particles' phase space density, $f(z, \mu, t)$, is described by the focused transport equation (Roelof 1969),

$\frac{\partial f}{\partial t}+v \mu \frac{\partial f}{\partial z}+\frac{1-\mu^{2}}{2 L} v \frac{\partial f}{\partial \mu}-\frac{\partial}{\partial \mu}\left(D_{\mu \mu} \frac{\partial f}{\partial \mu}\right)=q(z, \mu, t)$

where $t$ is the time, $z$ is the distance along the magnetic field line, and $\mu$ is the particle pitch-angle cosine. The focusing effect is characterized by the focusing length, $L(z)=-B(z) /(\partial B / \partial z)$, in the diverging magnetic field, $B$, while the pitch-angle diffusion coefficient, $D_{\mu \mu}$, describes stochastic forces. The injection of particles close to the Sun is given by $q(z, \mu, t)$. Equation (2) neglects convection and adiabatic deceleration (see Ruffolo 1995, for the full focused transport equation). As analytical solutions of the focused transport equation are not known, numerical model are developed. These models allow us to accurately fit in situ data and remove the uncertainties inherent in direct analysis methods, such as VDA and time-shifting analysis.

In this study, we make use of the results of the Monte Carlo interplanetary transport model developed by Agueda et al. (2008, 2012). The model assumes a static source of particles at two solar radii and an Archimedean spiral magnetic flux tube connecting the Sun and the spacecraft. The value of the solar wind speed measured by the spacecraft is used to estimate the curvature of the Parker spiral and constrain the global scenario. A turbulent component is assumed to be superposed onto the large structure of the IMF. Various pitch-angle diffusion models have been suggested to parametrize the wave-particle interactions, based on the original results of the classical quasi-linear theory (QLT) of particle scattering (Jokipii 1966) and improved later approaches (e.g. Beeck \& Wibberenz 1986). In this work, we parametrize the pitch-angle diffusion coefficient as in Agueda et al. (2008) and assume a form of the coefficient that resembles the results of the standard QLT by assuming that the spectral slope of the magnetic field power spectrum is $q=1.66$ (see Agueda \& Vainio 2013, for details). No variation across the magnetic field is assumed; that is, diffusion perpendicular to the average magnetic field is neglected. The radial mean free path, $\lambda_{r}$, is then the only free parameter that describes the amount of pitchangle scattering processes undergone by the energetic particles.

The interplanetary transport model provides us with the response of the system to an impulsive (delta) injection at the Sun, which is the Green's function of particle transport. Particle intensities measured in the heliosphere as a function of time, energy, and direction are obtained as a temporal convolution of the source function (particle release profile) and the Green's function of particle transport at the spacecraft location. The sources of SEP events can be unfold by solving the inverse problem (deconvolving the in situ measurements). Then one uses the measurements to infer the actual values of the model parameters. It is a deductive approach; it has the advantage that a systematic exploration of the parameters' space is possible, and the source function does not need to be parametrized a priori (Agueda et al. 2008). The problem is well constrained (the number of degrees of freedom is larger than the number of model parameters) if one uses the observed directional intensities to compare with the simulation results. If the directional information in the data is scarce or one only uses the omni-directional intensities to fit the event, then the problem is ill-posed; that is, multiple combinations of source and transport scenarios can provide an explanation to the observational data (Agueda et al. 2009b). 
Table 1. Observational characteristics of the selected NR electron events measured by ACE (62-102 keV) and Wind (50-82 keV).

\begin{tabular}{|c|c|c|c|c|c|c|c|c|c|c|c|c|c|}
\hline & \multirow[b]{2}{*}{ Date } & \multirow[b]{2}{*}{ DOY } & \multicolumn{5}{|c|}{ 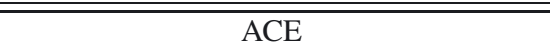 } & \multicolumn{5}{|c|}{ Wind } & \multirow{2}{*}{$\begin{array}{c}\mathrm{S} / \mathrm{C} \\
\text { Separation } \\
\left(R_{\oplus}\right) \\
\end{array}$} \\
\hline & & & $\begin{array}{c}t_{\oplus} \\
(\mathrm{UT})\end{array}$ & $\begin{array}{r}\text { Rise } \\
(\min )\end{array}$ & $\begin{array}{c}\langle u\rangle \\
\left(\mathrm{km} \mathrm{s}^{-1}\right)\end{array}$ & $\begin{array}{r}\text { Foot. } \\
\left({ }^{\circ}\right)\end{array}$ & $\begin{array}{l}\text { IMF } \\
\text { Pol. }\end{array}$ & $\begin{array}{c}t_{\oplus} \\
(\mathrm{UT})\end{array}$ & $\begin{array}{r}\text { Rise } \\
(\min )\end{array}$ & $\begin{array}{c}\langle u\rangle \\
\left(\mathrm{km} \mathrm{s}^{-1}\right)\end{array}$ & $\begin{array}{r}\text { Foot. } \\
\left({ }^{\circ}\right)\end{array}$ & $\begin{array}{l}\text { IMF } \\
\text { Pol. }\end{array}$ & \\
\hline \multirow{4}{*}{ 官 } & 1999 Jun. 11 & 162 & $00: 50$ & 45 & 445 & W55 & -1 & $00: 59$ & 37 & 455 & W53 & -1 & 60 \\
\hline & 2002 Feb. 20 & 51 & 06:00 & 24 & 398 & W61 & +1 & 06:09 & 22 & 412 & W59 & +1 & 340 \\
\hline & 2002 Dec. 19 & 353 & $21: 55$ & 24 & 530 & W46 & +1 & $22: 05$ & 16 & 550 & W44 & +1 & 132 \\
\hline & 2004 Nov. 1 & 306 & 06:05 & 29 & 419 & W58 & +1 & $06: 14$ & 27 & 431 & W56 & +1 & 42 \\
\hline \multirow{3}{*}{$\begin{array}{l}\text { ज } \\
\vdots \\
0 \\
0\end{array}$} & 2000 Sep. 12 & 256 & $12: 30$ & 619 & 372 & W65 & -1 & $12: 44$ & 589 & 370 & W66 & -1 & 274 \\
\hline & 2002 Jul. 7 & 188 & $11: 44$ & 286 & 415 & W59 & -1 & $11: 58$ & 378 & 424 & W57 & -1 & 282 \\
\hline & 2002 Aug. 14 & 226 & 01:49 & 35 & 430 & W57 & +1 & 02:00 & 35 & 443 & W55 & +1 & 208 \\
\hline
\end{tabular}

\section{Event selection}

The goal was to identify a comprehensive sample of NR electron events for which the inversion methodology could be applied. The SEP events best suited for simulation-based analyses are those that are best observed; that is, prominent SEP events, not masked by the pre-event background, and with good observational coverage of the pitch-angle distributions (PADs) to constrain the simulation-based analysis results. In addition, SEP events need to occur during nearly idealized conditions regarding the interplanetary scenario. As mentioned in Sect. 3.2, many interplanetary transport models assume an Archimedean spiral connecting the Sun and the observer. In that sense, it is important that selected SEP events occur during quiet interplanetary conditions, in the absence of interplanetary CMEs between the Sun and the observer or beyond. The SEP events should show velocity dispersion at the onset, which probes the fact that the intensity profiles observed in situ are not a result of a local effect.

Our initial sample was the SEPServer catalogue consisting of 115 proton events observed by SOHO/ERNE (Vainio et al. 2013). We considered the following selection criteria: 1) quietness in the interplanetary medium, which implies no interplanetary CME structure in the spacecraft nearby space within \pm 3 days of the event onset time; 2) significant enhancement of NR electron intensities, which implies a peak intensity at least an order of magnitude above the pre-event background in the 102-175 keV energy channel; 3) velocity dispersion at the onset of the event; and 4) good observational coverage of the PADs.

The first criterion was the most restrictive one, discarding more than half of the events in the initial sample. The second criterion, importance of the event, led us to discard about two dozens of events. By inspecting the normalized time intensity profiles of the remaining sample, we found five events that did not show dispersion in velocity at the onset. Finally, one of the remaining events exhibited low pitch-angle coverage (Agueda et al. 2009b) for ACE/EPAM observations. In the end, only a total of $11 \mathrm{NR}$ electron events in the initial sample fulfilled the criteria enumerated above.

Using Wind/3DP observations, we examined the evolution of the electron PADs for this sample of events and found two main groups. On one hand, seven events showed a monotonic evolution of the PADs, signaling particles streaming mainly away from the Sun and gradually isotropizing due to interplanetary scattering. On the other hand, four events exhibited PADs with changes in their topology during the first hours of the event. These changes (polarity reversals and/or the observation of bidirectional streams) were observed in most cases in coincidence with changes in the IMF direction. Agueda \& Klein (2013) analyzed one of the events in this sub-sample, occurring on 1998
April 20. The changes in the topology of the PADs may be related to flux tube variations or could be due the global scenario for these events, which was more complicated than the one assumed in the interplanetary transport model used in this study (see Agueda et al. 2010; Kartavykh et al. 2013, for additional examples). We discarded these four events from further analysis.

The dates of the sample of seven events selected for this study are listed in Table 1 (first and second columns). The third and fourth columns list the time of the onset of the event, $t_{\oplus}$, recorded in the E'2 (62-102 keV) energy channel by ACE and the event rise time. The following three columns give the mean value of the solar wind speed measured in situ, the longitude of the corresponding nominal footpoint of the IMF line connecting ACE with the Sun, and the IMF polarity. The same quantities (onset, rise time, solar wind speed, footpoint, and IMF polarity) are listed for Wind observations. The onset of the event observed by Wind is determined in the $50-82 \mathrm{keV}$ energy channel. The last column lists the ACE-Wind spacecraft separation (in Earth radii) for each event.

In all seven cases, the location of the ACE and Wind spacecraft were very similar (separation $<350 R_{\oplus}$ ), as well as the solar wind speed values measured in situ. The nominal footpoints of the spacecraft were approximately between W45 and W65 (solar wind speeds between 550 and $370 \mathrm{~km} \mathrm{~s}^{-1}$ ), and the IMF polarity was the same at both spacecraft. The event onset time was taken as the first time when the electron flux exceeded the preevent mean flux by at least three standard deviations. The event peak intensity was observed less than 45 min after the event onset time in 5 out of 7 events; in the other two events, the rise time was longer than four hours.

We classified the events in two groups, depending on the time over which the event PADs remained highly anisotropic, indicating a clear antisunward beaming of electrons. These times were inferred based on qualitative assessments of color-coded PADs of the $50-82 \mathrm{keV}$ electrons observed by Wind/3DP. We found that the first four events (Group I) in Table 1 showed nearly isotropic PADs in $<1 \mathrm{~h}$, while the remaining three events (Group II) showed highly anisotropic PADs for $\geq 2 \mathrm{~h}$.

Table 2 summarizes the properties of the electromagnetic emissions observed in association with each of the events. The first column gives the date of the event. Columns 2 to 4 give the characteristics of the SXR flare (start time, rise time, and class). Column 5 gives the location of the $\mathrm{H} \alpha$ flare. Columns 6 lists the start time and end time of the HXR emission. Columns $7-8$ give the estimated duration of the associated type III and type II radio bursts. Columns 9-13 list the parameters of the associated CMEs as reported in the $\mathrm{SOHO} / \mathrm{LASCO} \mathrm{CME}$ catalog: the time and height of the $\mathrm{CME}$ at the first appearance in the $\mathrm{C} 2$ coronagraph, the position angle (PA, measured counterclockwise from the 
Table 2. Electromagnetic emissions associated with the electron events.

\begin{tabular}{|c|c|c|c|c|c|c|c|c|c|c|c|c|c|}
\hline & \multirow{4}{*}{ Date } & \multicolumn{6}{|c|}{ Solar flare } & \multirow{5}{*}{$\begin{array}{l}\text { DH Type II } \\
\text { Time } \\
\text { (UT) } \\
\text { (8) }\end{array}$} & \multirow{2}{*}{\multicolumn{5}{|c|}{ CME }} \\
\hline & & \multicolumn{3}{|c|}{ Soft X-rays } & \multirow{4}{*}{$\begin{array}{c}\mathrm{H} \alpha \\
\text { Position } \\
(5)\end{array}$} & \multirow{4}{*}{$\begin{array}{c}\text { Hard X-rays } \\
\text { Time } \\
\text { (UT) } \\
\text { (6) }\end{array}$} & \multirow{4}{*}{$\begin{array}{l}\text { Type III } \\
\text { Time } \\
\text { (UT) } \\
\text { (7) }\end{array}$} & & & & & & \\
\hline & & Start & Rise & X-ray & & & & & \multicolumn{2}{|c|}{ First Ob. } & $\overline{\mathrm{PA}}$ & Speed & \multirow{3}{*}{$\begin{array}{c}\text { Width } \\
\left({ }^{\circ}\right) \\
(13)\end{array}$} \\
\hline & & (UT) & $(\min )$ & class & & & & & $t(\mathrm{UT})$ & $r\left(R_{\odot}\right)$ & $\left({ }^{\circ}\right)$ & $\left(\mathrm{km} \mathrm{s}^{-1}\right)$ & \\
\hline & (1) & (2) & (3) & (4) & & & & & (9) & (10) & (11) & (12) & \\
\hline \multirow{11}{*}{ 官 } & 1999 Jun. 11 & 01:05 & 5 & $\mathrm{C} 1.0$ & $\sim \mathrm{W} 120$ & no RHESSI & $00: 40-00: 46$ & - & $01: 26$ & 4.78 & 288 & 719 & 101 \\
\hline & 2002 Feb. 20 & 05:52 & 20 & M5.1 & N12 W72 & $06: 16-06: 42$ & 05:53-06:39 & - & $06: 30$ & 3.46 & Halo & 952 & 360 \\
\hline & & 07:41 & 3 & $\mathrm{C} 2.5$ & N17 W81 & 08:03-08:24 & 08:07-08:20 & & & & & & \\
\hline & & 09:46 & 13 & M4.3 & N18 W83 & 09:46-10:01 & 09:55-10:07 & & & & & & \\
\hline & & & & & & $10: 19-10: 23$ & $10: 18-10: 25$ & & & & & & \\
\hline & & 11:02 & 5 & C7.5 & N15 W77 & 11:04-11:06 & $11: 03-11: 20$ & & & & & & \\
\hline & 2002 Dec. 19 & $21: 34$ & 19 & M2.7 & N23 W21 & $21: 32-21: 47$ & $21: 35-21: 59$ & $21: 45-22: 30$ & 22:06 & 4.20 & Halo & 1029 & 360 \\
\hline & & & & & N15 W09 & $23: 10-23: 45$ & $23: 03-23: 15$ & & & & & & \\
\hline & & $00: 32$ & 5 & $\mathrm{C} 4.5$ & S25 W27 & $23: 47-23: 51$ & $23: 50-24: 00$ & & & & & & \\
\hline & 2004 Nov. 1 & 06:14 & 5 & B5.4 & >W90 & 05:28-05:30 & $05: 23-06: 43$ & $05: 55-07: 25$ & 06:06 & 2.98 & 266 & 925 & 146 \\
\hline & & 06:55 & 7 & $\mathrm{C} 2.9$ & & & & & & & & & \\
\hline \multirow{7}{*}{ 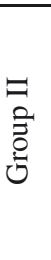 } & 2000 Sep. 12 & $11: 31$ & 42 & M1.0 & S17 W09 & no RHESSI & $11: 43-12: 20$ & 12:00-15:00 & $11: 54$ & 2.83 & Halo & 1550 & 360 \\
\hline & 2002 Jul. 7 & $11: 15$ & 28 & M1.0 & $\sim$ W95 & $11: 30-11: 38$ & $11: 14-11: 21$ & $11: 35-20: 00$ & $11: 30$ & 4.37 & 277 & 1423 & $>228$ \\
\hline & & & & & & & $11: 39-11: 49$ & & & & & & \\
\hline & & & & & & & $12: 41-12: 52$ & & & & & & \\
\hline & 2002 Aug. 14 & 01:47 & 25 & M2.3 & N09 W54 & 02:14-03:15 & $01: 37-02: 18$ & $02: 20-24: 00$ & $02: 30$ & 5.55 & 297 & 1309 & 133 \\
\hline & & & & & & 02:34-02:38 & 02:59-03:09 & & & & & & \\
\hline & & & & & & & $04: 18-04: 25$ & & & & & & \\
\hline
\end{tabular}

conventional solar north), the plane-of-sky speed of the leadingedge and the angular width.

Group I (1999 Jun. 11, 2002 Dec. 19, 2002 Feb. 20 and 2004 Nov. 1) was associated with impulsive SXR flares with rise times $\leq 20 \mathrm{~min}$. For two of the events (1999 Jun. 11 and 2004 Nov. 1), the associated flare occurred behind the west limb. This is shown by the lack of SXR flare at the time of the type III radio burst, the weak high frequency emission of the DH type III radio burst, and the lack of brightening in EIT difference images. The other two events (2002 Dec. 19 and 2002 Feb. 20) were associated with large flares in the western hemisphere of the Sun, followed by later SXR flares and type III radio bursts during the next four hours. CMEs were observed for all the events, with plane-of-sky speeds between 700 and $1000 \mathrm{~km} \mathrm{~s}^{-1}$. Two CMEs appeared as halo CMEs, and the other two had widths of $\sim 100-150^{\circ}$. For some of the events (2/4), type II radio bursts were reported with a broad diffusive band (2002 Dec. 19) and type III defined tones (2004 Nov. 1).

Group II (2000 Sep. 12, 2002 Jul. 7 and 2002 Aug. 14) was associated with gradual SXR flares with rise times larger than $25 \mathrm{~min}$ and long SXR decay times. The associated flares were larger than M1.0 and distributed across the west hemisphere of the Sun (central meridian, nominal footpoint, and beyond the west limb). Unfortunately, the peak in HXR emission was missed by RHESSI for those events when data was available. Type III radio bursts were observed after the onset of the SXR emission. For the 2002 Aug. 14 event, the relevant type III group was not easy to identify due to a type III storm, but long decay microwave emission $(5 \mathrm{GHz})$ was observed, as well as further faint and isolated type III bursts. Type II radio bursts were reported on the Wind/WAVES website. They included intense broad band (2000 Sep. 12), intermittent (2002 Jul. 7) and broad band (2002 Aug. 14). During the 2000 Sep. 12, Nançay Radioheliograph observations showed a type IV burst signaling time-extended electron acceleration in the corona. Fast $\left(>1300 \mathrm{~km} \mathrm{~s}^{-1}\right.$ ) CMEs were observed for all the events. The CME observed in association with the central meridian flare (2000 Sep. 12; W09) appeared as a halo CME, and the other two had widths $>130^{\circ}$ and appeared in the western hemisphere of the Sun.

\section{Results}

We used the SEPinversion software developed within the FP7 project SEPServer ${ }^{3}$ to infer the release time history and the interplanetary transport conditions of NR electrons for each event. SEPinversion is an IDL-based software, freely available online ${ }^{4}$, based on the work by Agueda et al. $(2008,2012)$.

In this study, we employed NR electron data by ACE and Wind covering the approximate energy range between 50 and $300 \mathrm{keV}$ in three energy channels. To find out the best fit scenario, we considered a wide range of interplanetary transport conditions covering 20 values of the radial mean free path, logarithmically spaced between 0.05 to $1.20 \mathrm{AU}$. For each transport scenario, the best possible release time history was obtained. The goodness of the fit for each case was then evaluated by comparing the observations and the modeled data (see Agueda et al. 2008, 2009a, for more details). Each energy channel was fitted separately and the goodness-of-fit estimator of the whole fit was obtained as a sum of the goodness-of-fit values obtained for each energy channel.

Figure 1 and 2 show the results for events in Group I, and Figs. 3 and 4 show the results for events in Group II. For each plot, the two top panels (A and B) show EM emissions observed in association with the event, that is, SXR flux observed by GOES and HXR emission detected by RHESSI whenever available, radio flux observed by Wind/WAVES, and height-time plot of the CME leading edge observed by SOHO/LASCO. Panel C shows the release time history at two solar radii inferred using SEPinversion. The profile has been shifted by +500 s to allow comparison with the electromagnetic emissions. The dashed area

\footnotetext{
3 http://wwww.sepserver.eu/

4 http://server.sepserver.eu/
} 

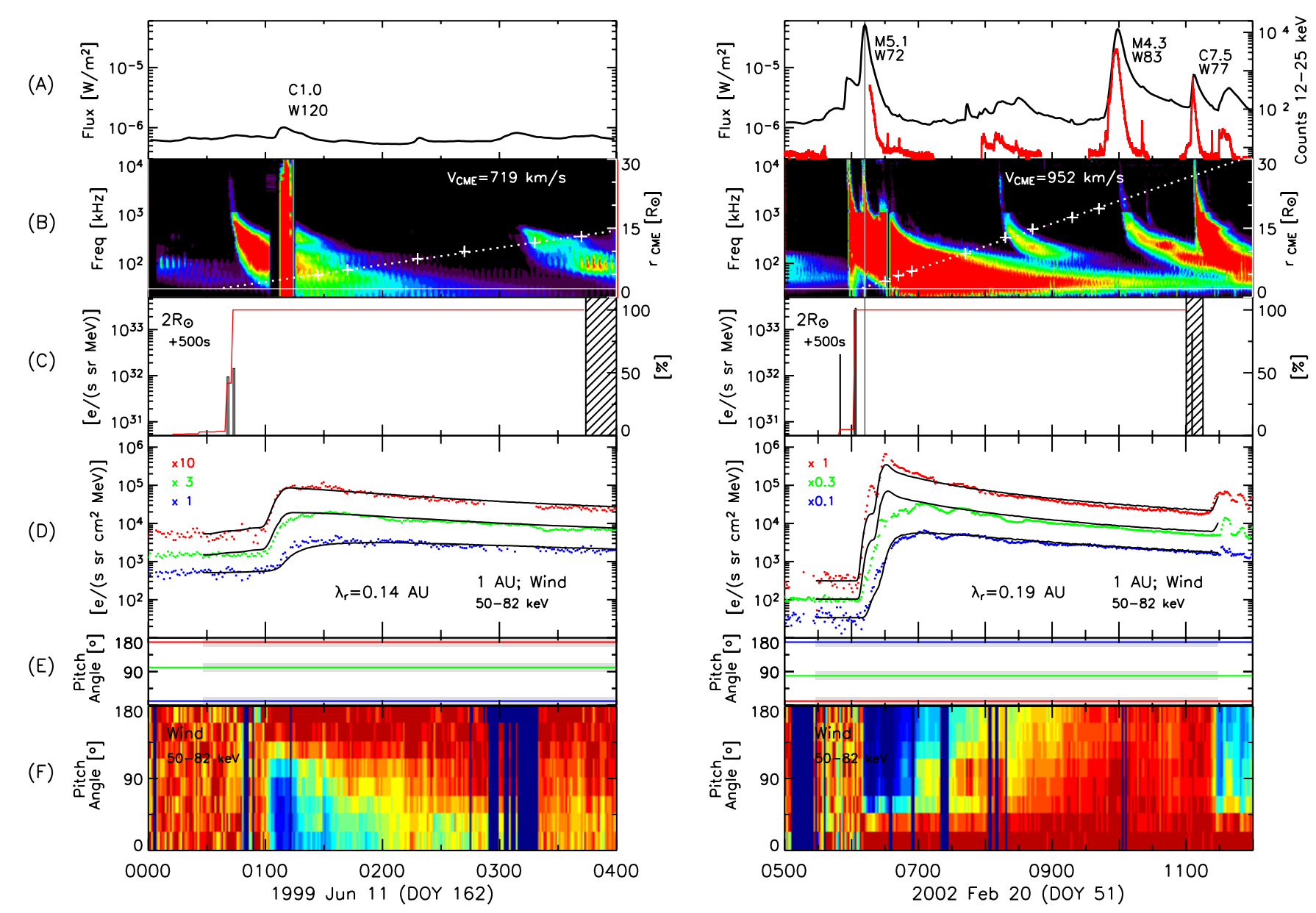

Fig. 1. From top to bottom and for each event: (A) soft X-ray flux observed by GOES and hard X-ray emission detected by RHESSI (red curve; right axis) when available. (B) Radio flux observed by Wind/WAVES (the white horizontal line marks $30 \mathrm{kHz}$ ) and time-height plot of the CME leading edge (dotted curve; right axis). (C) Electron source profile deduced at $2 R_{\odot}$ (gray histogram) and total percentage of electrons (red curve; right axis). The profile has been shifted by $+500 \mathrm{~s}$ to allow the comparison with the EM emissions. (D) Directional NR electron intensities measured near 1 AU. (E) Mean pitch-angle and range (gray area) scanned by each sector. (F) PADs measured by Wind/3DP normalized to unity in each time interval.

in panel $\mathrm{C}$ marks the time interval when the results of the inversion should be taken with care, as release episodes occurring during this period produce a response at the spacecraft location peaking outside the fitting period. Panel $\mathrm{C}$ also shows the release percentage of electrons over the total; release episodes occurring within the dashed area were omitted in the computation. The next two panels (D and E) show the observed directional intensities. For simplicity, panel D only shows three directional intensity profiles, and panel E displays the associated pitch-angles. Data is colored, and the results of the fit are shown in black. Although not displayed, we used the maximal amount of directional information of the particle distribution present in the data for fitting in all cases. We used the eight sectored intensities observed by the LEFS60 telescope on board ACE/EPAM and, when available, the PADs computed by Wind/3DP in eight pitch-angle bins.

To provide a general overview of the evolution of the PADs of the event, we included the 50-82 keV electron PADs recorded by Wind in panel F. These plots are normalized to unity in each time interval. They show isotropic distributions during the preevent background period and anisotropic distributions when the event starts. We note that when the event starts the sunward part of the distribution is very small compared to the antisunward part; thus, the reader should not interpret the drop of intensity in the PADs plots as a sign that the intensities do decrease in absolute values. It can be seen that four of the events exhibit large data gaps in Wind observations (dark blue areas). For these events, we only inverted ACE observations.

\subsection{Interplanetary transport conditions}

Table 3 shows the values of the radial mean free path that provide the best fit for each event. The first column lists the date of the event, the second column lists the spectral index of the source, and the next eight columns list the best fit $\lambda_{r}$-values obtained for each energy channel, using either ACE or Wind observations, and the $\lambda_{r}$ values providing the best global fit.

In the interplanetary transport simulations, $\lambda_{r}$ is assumed to be energy independent over the energy range under study. If we take the value of $\lambda_{r}$ which provides the best fit in all fitted energy channels, the obtained values range from $0.12 \mathrm{AU}$ to $0.44 \mathrm{AU}$. When both ACE and Wind observations were fitted, the inferred values of $\lambda_{r}$ were very similar (within two consecutive values in the tested radial mean free path grid). Figures 1-4 display the observed $\sim 50-100 \mathrm{keV}$ electron intensities (colored curves) and modeled intensities (black curves). It can be 


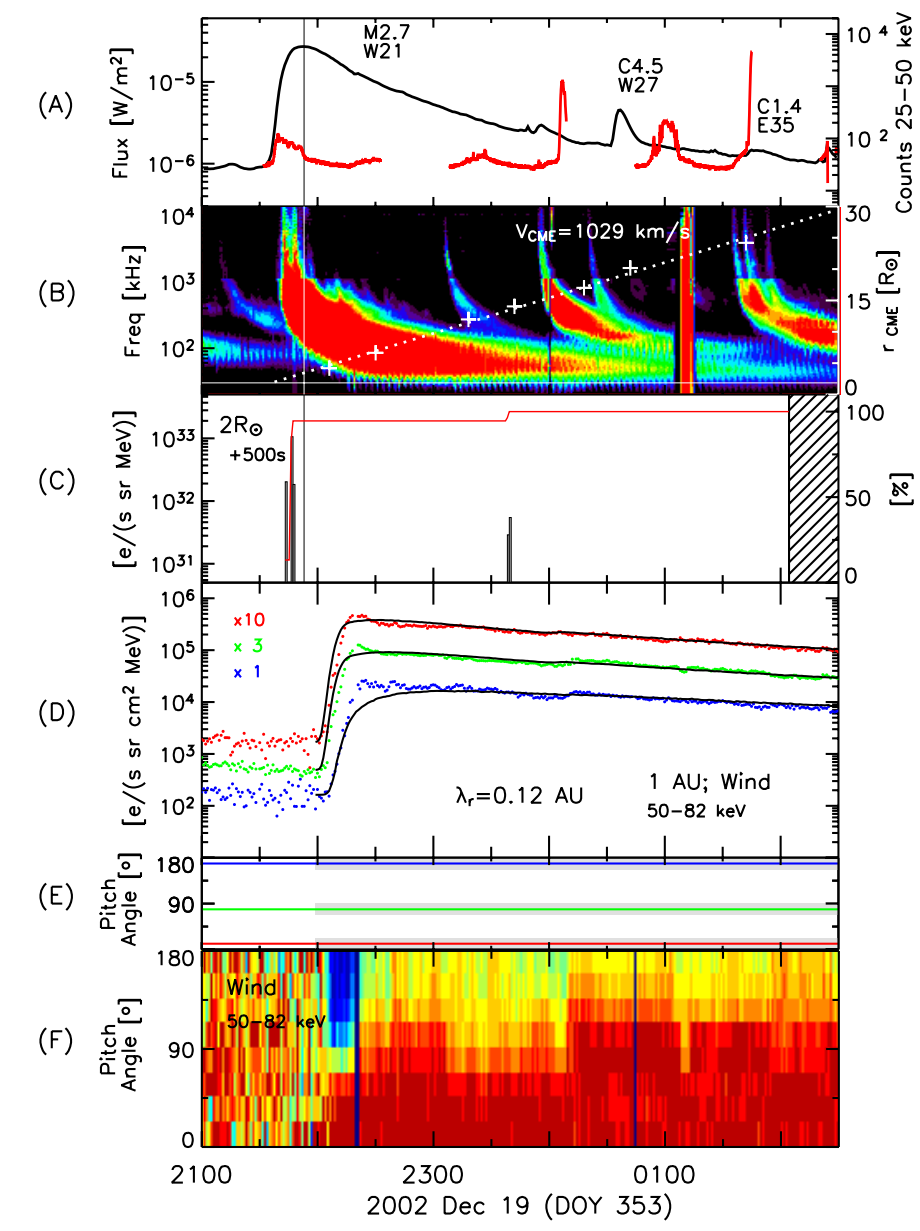

Fig. 2. Same format as in Fig. 1.

seen that the observational profiles are well reproduced by the modeling results; that is, the evolution of the PADs is explained during $\geq 4 \mathrm{~h}$.

If we allowed the value of the mean free path to vary from one energy channel to the other, the trend in Table 3 would suggest that the best fit case is obtained for slightly larger values of $\lambda_{r}$ for the lowest energy channel, and lower values of $\lambda_{r}$ for the highest energy channel.

\subsection{Release timescales compared with electromagnetic emissions}

We found two types of release time histories. Events in Group I show a prompt release (see Figs. 1 and 2) lasting less than $60 \mathrm{~min}$. At low energies $(50-82 \mathrm{keV}$ or $62-102 \mathrm{keV}$, depending on the observations), the timing of the first particle release agrees with the timing of the type III radio burst within $5 \mathrm{~min}$. Moreover the duration of the release is consistent with the duration of the main type III burst of the period. This is observed even for the two events associated with an occulted active region behind the west limb. For two events (2002 Dec. 19 and 2002 Feb. 20), we note that no release is observed in association with later type III radio bursts. It is important to note that these bursts do not reach frequencies at or below $\sim 30 \mathrm{kHz}$, which corresponds to the quasi-thermal noise emission extending across the dynamic spectra induced by the motion of ambient electrons in the vicinity of the spacecraft, also known as plasma line (e.g. Meyer-Vernet \& Perche 1989; Stone et al. 1992). Thus, the

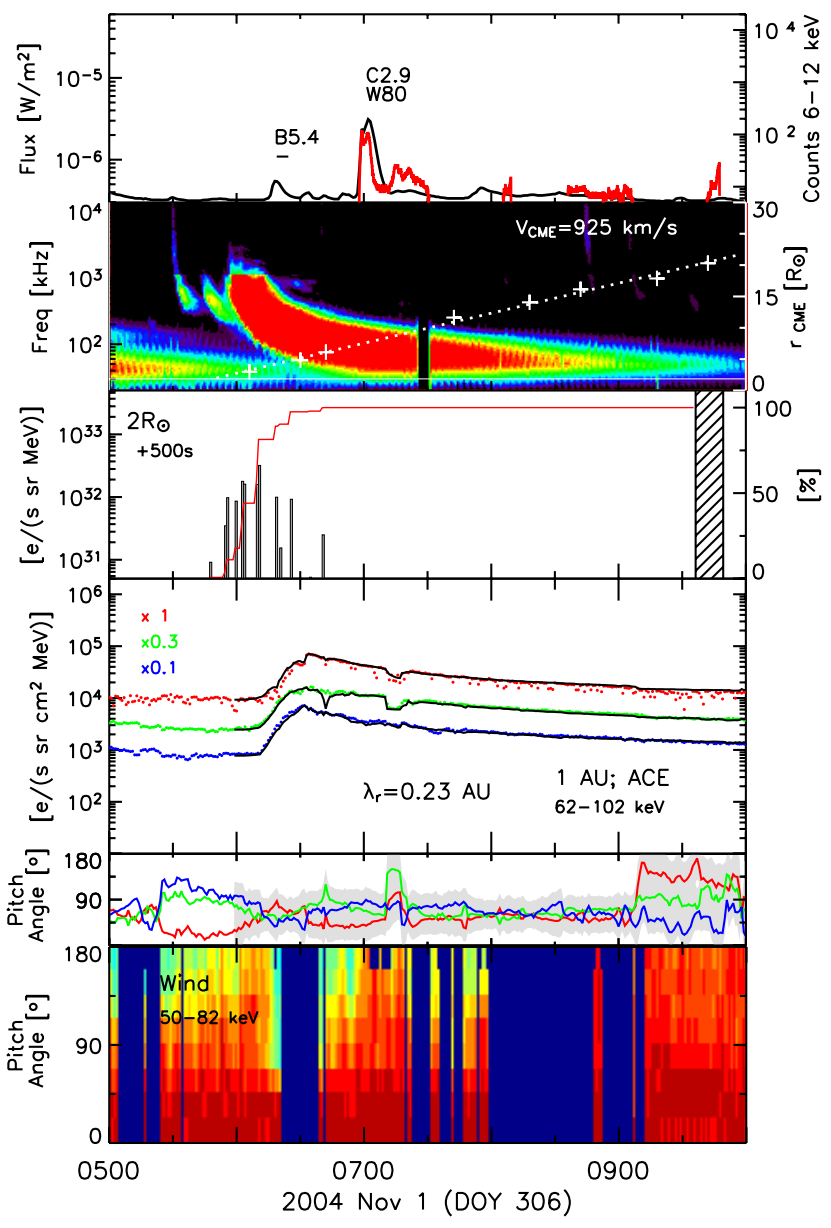

source/s releasing the later type III emitting electron beams was/were not magnetically connected to the spacecraft.

Events in Group II exhibit release time histories lasting several hours (see Figs. 3 and 4). For two of the events (2000 Sep. 12 and 2002 Jul. 7), the inferred particle release began between 20 and 45 min after the beginning of the type III radio emission, and the type III radio bursts did not reach the local plasma frequency. The electron release started in coincidence with the type III radio bursts on 2002 Aug. 14, but the duration of the particle release appeared to be sustained during $>3 \mathrm{~h}$, which is well after the end of the type III radio emission. Signatures of long-duration acceleration processes in the corona were observed for events in Group II. In particular, long decay microwave emission $(5 \mathrm{GHz})$ was observed on 2002 Aug. 14, a type IV burst was observed by the Nançay Radioheliograph on 2000 Sep. 12 (Klein et al. 2005) and type II radio bursts were reported on the three dates. CMEs with large plane-of-sky speeds $\left(>1300 \mathrm{~km} \mathrm{~s}^{-1}\right)$ were observed for all the events in Group II, with larger speeds than the CMEs in Group I.

Table 4 lists the release timings inferred from the VDA and the timings obtained using simulation-based methods. The first column lists the date of the event. The second and third columns list the release start time, $t_{0}$, and the proton apparent path length, $L$, derived by Vainio et al. (2013) using 1-131 MeV proton intensities measured by SOHO/ERNE. The fourth and fifth columns list the electron release start time and the apparent path length derived in this study using Wind/3DP observations. For two sets of observations (62-102 keV electrons observed by ACE and 50-82 keV electrons observed by Wind), the following columns 

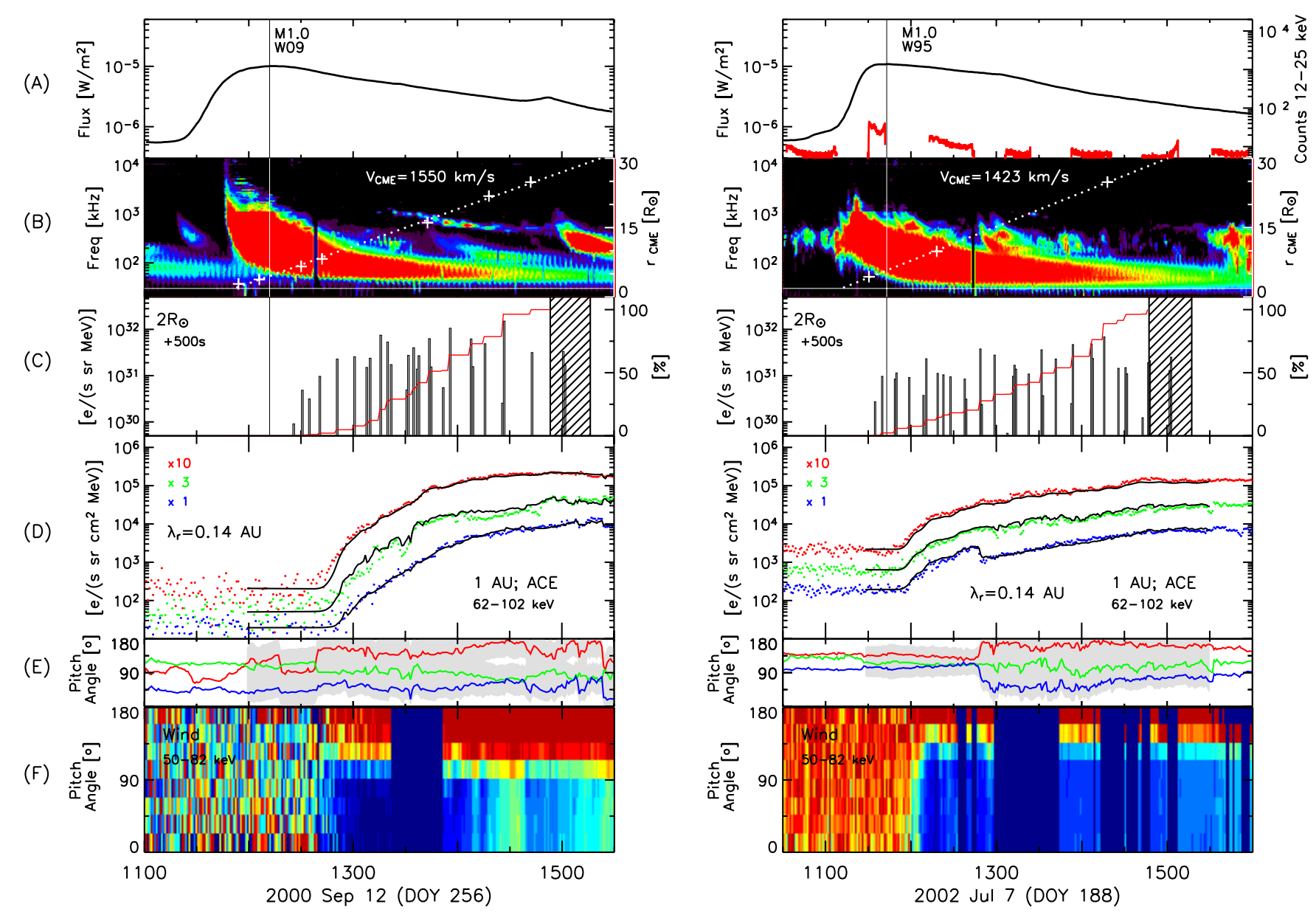

Fig. 3. Same format as in Fig. 1.

Table 3. Electron radial mean free path providing the best fit for each event, using ACE and Wind observations.

\begin{tabular}{|c|c|c|c|c|c|c|c|c|c|c|}
\hline & \multirow[b]{3}{*}{ Event } & \multirow[b]{3}{*}{$\gamma$} & \multicolumn{8}{|c|}{ Electron radial mean path (AU) } \\
\hline & & & \multicolumn{4}{|c|}{ ACE observations } & \multicolumn{4}{|c|}{ Wind observations } \\
\hline & & & $175-312 \mathrm{keV}$ & $102-175 \mathrm{keV}$ & $62-102 \mathrm{keV}$ & Total & $135-230 \mathrm{keV}$ & $82-135 \mathrm{keV}$ & $50-82 \mathrm{keV}$ & Total \\
\hline \multirow{4}{*}{ 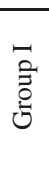 } & 1999 Jun. 11 & 2.5 & 0.16 & 0.16 & 0.16 & 0.16 & 0.14 & 0.14 & 0.16 & 0.14 \\
\hline & 2002 Feb. 20 & 2.5 & 0.23 & 0.27 & 0.37 & 0.27 & 0.16 & 0.19 & 0.23 & 0.19 \\
\hline & 2002 Dec. 19 & 2.5 & 0.10 & 0.10 & 0.12 & 0.12 & 0.10 & 0.12 & 0.14 & 0.12 \\
\hline & 2004 Nov. 1 & 2.5 & 0.14 & 0.19 & 0.27 & 0.23 & \multicolumn{4}{|c|}{ Long data gaps } \\
\hline \multirow{3}{*}{$\begin{array}{l}\text { ज } \\
\vdots \\
0 \\
0 \\
0\end{array}$} & 2000 Sep. 12 & 2.5 & 0.12 & 0.14 & 0.16 & 0.14 & \multicolumn{4}{|c|}{ Long data gaps } \\
\hline & 2002 Jul. 7 & 2.0 & 0.12 & 0.14 & 0.19 & 0.14 & \multicolumn{4}{|c|}{ Long data gaps } \\
\hline & 2002 Aug. 14 & 3.5 & 0.31 & 0.44 & 0.52 & 0.44 & \multicolumn{4}{|c|}{ Long data gaps } \\
\hline
\end{tabular}

list the start time of the release time history, $t_{0}$, the estimated duration, $\Delta t$, the amount of released electrons and the value of the interplanetary mean free path, $\lambda_{r}$.

It can be seen from Table 4 that the release start time inferred using a simulation-based analysis occurs within 15 min of the release start time obtained with a VDA of ERNE observations in all cases. The release start time obtained with a VDA of Wind observations is always delayed with respect to the release start time inferred by using a simulation-based analysis, with time differences that range from 15 to $35 \mathrm{~min}$. The values of the apparent path length obtained with a VDA of ERNE observations do not show a clear correlation with the values of the mean free path obtained from the modeling of the electron events. Most of the values of the apparent path length obtained with a VDA of Wind observations are unphysical (smaller than 1 AU), highlighting the fact that a simple VDA of these observations does not provide reliable results and that the effects of pre-event background and onset contamination by high energy electrons might be an issue during the rising phase of these events.

The amount of NR electrons released in the interplanetary medium range from $\sim 10^{33}$ to $10^{35} \mathrm{e} \mathrm{sr}^{-1} \mathrm{MeV}^{-1}$ (see Table 4). The values inferred using ACE and Wind observations for the same date are consistent within a factor of 3 . The mean injection rate for long release time histories (Group II) is smaller than the mean injection rate deduced for events in Group I, but the release is more sustained in time, which makes it possible to deliver a larger amount of particles in the spacecraft flux tube. 
Table 4. Release times inferred from the VDA of proton ERNE observations and Wind/3DP electron observations with the results of the inversion of in situ electron directional intensities.

\begin{tabular}{|c|c|c|c|c|c|c|c|c|c|c|c|c|c|}
\hline & \multirow[b]{3}{*}{ Date } & \multirow{2}{*}{\multicolumn{2}{|c|}{$\begin{array}{l}\text { Proton } \\
\text { VDA }\end{array}$}} & \multirow{2}{*}{\multicolumn{2}{|c|}{$\begin{array}{c}\text { Electron } \\
\text { VDA }\end{array}$}} & \multicolumn{8}{|c|}{ Inversion of in situ electron obs. } \\
\hline & & & & & & \multicolumn{4}{|c|}{ ACE $62-102 \mathrm{keV}$} & \multicolumn{4}{|c|}{ Wind $50-82 \mathrm{keV}$} \\
\hline & & $\begin{array}{c}t_{0} \\
(\mathrm{UT})\end{array}$ & $\begin{array}{c}L \\
(\mathrm{AU})\end{array}$ & $\begin{array}{c}t_{0} \\
(\mathrm{UT})\end{array}$ & $\begin{array}{c}L \\
(\mathrm{AU})\end{array}$ & $\begin{array}{c}t_{0} \\
(\mathrm{UT})\end{array}$ & $\begin{array}{c}\Delta t \\
(\mathrm{~min})\end{array}$ & $\begin{array}{l}\text { Released Part. } \\
\left(\mathrm{e} \mathrm{sr}^{-1} \mathrm{MeV}^{-1}\right)\end{array}$ & $\begin{array}{c}\lambda_{r} \\
(\mathrm{AU})\end{array}$ & $\begin{array}{c}t_{0} \\
(\mathrm{UT})\end{array}$ & $\begin{array}{c}\Delta t \\
(\mathrm{~min})\end{array}$ & $\begin{array}{l}\text { Released Part. } \\
\left(\mathrm{e} \mathrm{sr}^{-1} \mathrm{MeV}^{-1}\right)\end{array}$ & $\begin{array}{c}\lambda_{r} \\
(\mathrm{AU})\end{array}$ \\
\hline \multirow{4}{*}{ 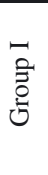 } & 1999 Jun. 11 & $00: 32 \pm 07$ & $1.81 \pm 0.09$ & $00: 58$ & 0.6 & $00: 39$ & 8 & $6.8 \times 10^{33}$ & 0.16 & $00: 40$ & 3 & $1.5 \times 10^{34}$ & 0.14 \\
\hline & 2002 Feb. 20 & $05: 44 \pm 06$ & $1.62 \pm 0.08$ & $06: 12$ & 0.3 & 05:51 & 30 & $1.1 \times 10^{35}$ & 0.27 & $05: 49$ & 14 & $3.5 \times 10^{35}$ & 0.19 \\
\hline & 2002 Dec. 19 & $21: 36 \pm 6$ & $1.61 \pm 0.07$ & 22:01 & 0.7 & $21: 26$ & 24 & $3.4 \times 10^{34}$ & 0.12 & $21: 43$ & 4 & $8.7 \times 10^{34}$ & 0.12 \\
\hline & 2004 Nov. 1 & $05: 42 \pm 6$ & $1.60 \pm 0.07$ & 06:02 & 1.2 & $05: 47$ & 53 & $7.7 \times 10^{34}$ & 0.23 & - & - & - & - \\
\hline \multirow{3}{*}{ 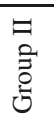 } & 2000 Sep. 12 & $12: 29 \pm 09$ & $1.76 \pm 0.12$ & $12: 41$ & 0.7 & $12: 25$ & 137 & $5.2 \times 10^{34}$ & 0.14 & - & - & - & - \\
\hline & 2002 Jul. 7 & $11: 26 \pm 04$ & $1.77 \pm 0.05$ & $11: 54$ & 0.7 & $11: 34$ & 187 & $3.1 \times 10^{35}$ & 0.14 & - & - & - & - \\
\hline & 2002 Aug. 14 & $02: 01 \pm 07$ & $1.29 \pm 0.08$ & 02:04 & 0.2 & $01: 46$ & 195 & $1.7 \times 10^{35}$ & 0.44 & - & - & - & - \\
\hline
\end{tabular}

Notes. All times have been shifted by +500 s to allow comparison with EM emissions.

(A)

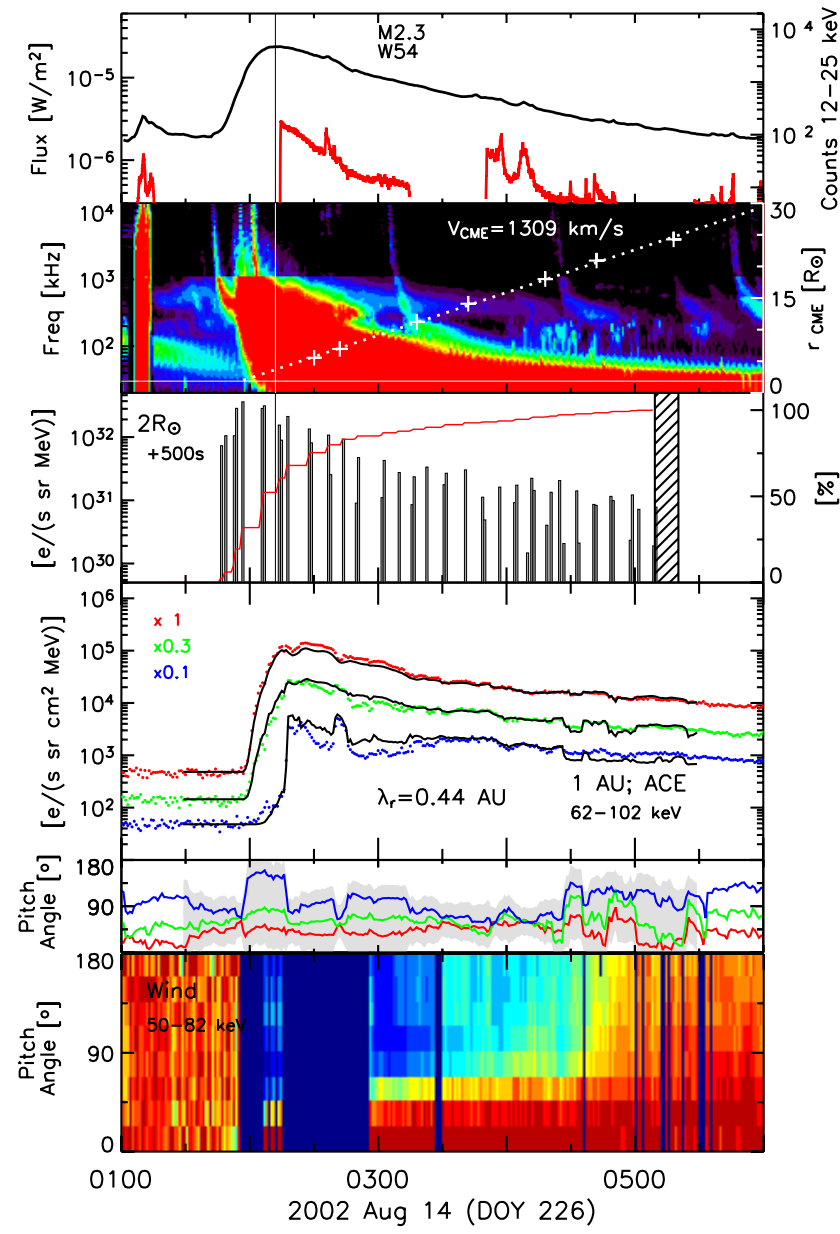

Fig. 4. Same format as in Fig. 1.

\section{Discussion}

SEPinversion constitutes a novel tool to study the solar sources of SEP events. This tool has been applied here to a systematic study of seven carefully selected electron events. The analysis tool is also available on the SEPServer web site.

\subsection{Caveats of the model and mitigation strategies}

It is worth to point out some of the limitations of the inversion methodology. First of all, it relies on the results of an interplanetary transport model that assumes that the large scale IMF can be described by an Archimedean spiral. Therefore, it is only be able to provide an explanation to SEP events occurring during quiescent interplanetary conditions. Another restriction to take into account is that the model assumes a fixed source of electrons located at two solar radii. If the source releasing particles to the interplanetary medium is moving (e.g. related to the expansion of a coronal shock), the model produces consistent results for NR electrons, but only if the study is restricted to the first hours of the event and as long as the SEPs travel the distance from two solar radii to the actual source location in a time interval smaller than the data time resolution. In addition, input measurements of directional intensities measured in a wide range of pitch-angles are required to constrain the inversion problem; otherwise, there is too much freedom in the data. That is, several combinations of release and transport scenarios can provide an explanation to the event.

The inversion methodology can also be limited by spurious release episodes, which may appear due to flux tube changes. For example, the release episode obtained around 23:30 UT on 2002 Dec. 19 (see Fig. 2) is related to the bump appearing in the particle data around 24 UT. The inversion procedure assumes that all changes in the intensity profiles are due to either transport or solar release processes, but a careful analysis of the observations reveals that this bump is associated with a change of the magnetic field direction observed in situ. Because we can still explain the event, it suggests that the transport conditions and the release time history in the neighboring flux tubes were similar. The spurious nature of the release episode is also revealed by its small magnitude ( $4 \%$ of the whole release profile) and the fact that it has no counterpart in the radio emission.

Other spurious release episodes may appear due to a large fluctuating pre-event background. In this case, the spurious episodes appear as precursors of the main release episode, and they also represent a very small fraction of particles in comparison to the rest of the profile. In that respect, identifying the amount of noise in the data can improve the quality of the inferred release time profile. Another thing to take into account is that the short gaps obtained for prolonged release time profiles 
should not be regarded as physical, but related to statistical fluctuations in the data that are not present in the Green's functions used for fitting the event. A smooth release time profile (with the same total amount of electrons) would provide indeed a very similar fit of the event.

\subsection{Impulsive and extended/sustained particle releases}

The results obtained for this set of well-selected events suggest that the release timescales of NR electrons in the low corona show a bimodal distribution. Short release episodes associated with type III radio emission (Group I) are obtained, suggesting that the NR electrons observed in interplanetary space were released at solar flares. It is commonly accepted that the speeds of type III-producing electron beams are about $0.1-0.2 c$ (kinetic energies of few to $10 \mathrm{keV}$; Aschwanden 2002), based on the speeds derived from the frequency drift of type III radio bursts. However, some observations (Poquérusse 1994) and recent simulation efforts (Li \& Cairns 2013) show that electron beams generating type III bursts may on occasion have much higher speeds, which agrees more with the energies analyzed in this study.

It is important to note that not every type III radio burst has an associated release episode. Only type III radio bursts extending down to the plasma line measured at the spacecraft have a release counterpart. This is consistent with a scenario where electrons released during type III radio bursts not reaching the local plasma line never reach the observer due to the lack of magnetic connectivity. Another interesting result is that connectivity does not seem to be problematic for events associated with occulted active regions (2002 Dec. 19 and 2004 Nov. 1), providing further evidence of the divergence of open magnetic flux in the low corona (Klein et al. 2008).

The source of sustained release obtained for events in Group II is harder to disentangle. On one hand, there are two events (2000 Sep. 12 and 2002 Jul. 7) for which the release time profile started well after the main type III radio burst, suggesting that the type III emitting electrons were missed by the spacecraft (also signaled by the fact that the radio emission did not extend down to the plasma line measured at the spacecraft). This was already reported by Klein et al. (2005) from the analysis of Wind/WAVES observations on 2000 Sep. 12. They found a southward trend of the centroid positions of the radio sources with decreasing frequency, suggesting that the bulk of the type III producing electron beams traveled far south of the ecliptic plane.

The inferred electron release time profile on 2000 Sep. 12 was accompanied by a type II radio burst observed by Wind/WAVES. The burst was readily visible in the spectrogram at and below $1 \mathrm{MHz}$ after 13:10 UT. At dm-m waves, timeextended electron acceleration in the corona was revealed by a type IV burst (Klein et al. 2005). The acceleration of electrons producing the long lasting meter wave emission can not be explained by a coronal shock, since the shock travels through the meter wave source in a time lapse much shorter than the event duration (Klein et al. 2005). Even when a type II burst demonstrates that a shock exists, a different mechanism of long lasting acceleration might thus operate in the corona in this event. The observation of type IV emission where post flare loops were observed, points to the importance of post-eruptive magnetic reconnection as a possible source of NR electrons observed in space (Klein et al. 2005).

The sustained release obtained for the event on 2002 Jul. 7 also starts after the bright type III radio burst. About an hour later, numerous DH type III bursts are observed starting near
$1 \mathrm{MHz}$, hence at rather low frequencies. This occurred during the long-lasting decay of the SXR burst, whose non-exponential evolution reveals further energy release after the peak. No clear HXR or meter wave radio emission is observed during this time that would point to electron acceleration in the low/middle corona. While the absence of HXR is expected from the occultation of the active region by the solar limb, there is also no radio evidence, such as type IV emission, of electron acceleration in the middle corona. A long-lasting type II burst was reported on 2002 Jul. 7, suggesting that the acceleration of NR electrons might be related to a coronal shock.

The time-extended release time profile inferred for the event on 2002 Aug. 14 shows the peculiarity that the start time appears in coincidence with the observed type III bursts, and the main type III burst does reach the plasma line at the spacecraft location. The most intense release is obtained during the type III radio burst, and it gradually decays providing a sustained release of particles for about $3 \mathrm{~h}$. A long-lasting microwave burst (not shown in Fig. 4) accompanies the particle release and when RHESSI starts observation at 02:14 UT, it has a high HXR count rate that subsequently decays.

All the selected events were associated with relatively fast CMEs $\left(>700 \mathrm{~km} \mathrm{~s}^{-1}\right.$ ) with widths (three halos and four CMEs with widths larger than $100^{\circ}$ ) suggesting that a large coronal volume was participating in the CME eruption. However, if the associated CMEs did drive shocks for events in Group I, they were not the sources of the escaping electrons detected in situ. Another possibility is that they provided particles into different magnetic structures that were not connected to the L1 spacecraft. The events exhibiting long sustained release time profiles were associated with the largest CME speeds $\left(>1300 \mathrm{~km} \mathrm{~s}^{-1}\right)$. If the source of these events is related to coronal shocks, the source propagation beyond $\sim 30 R_{\odot}$ could affect the timing of the release time profile (as a static source is assumed in the analysis). However, the transport of NR electrons beyond the source region would not be affected, as for all our events, when the electron mean free path is larger than the focusing lengths in the corona.

\subsection{The influence of interplanetary scattering}

Dröge (2003) presented a dynamical scattering model, including the effects of the dissipation range and resonance broadening, that provided an explanation to the previously observed rigidity dependence of particle mean free paths (Dröge 2000). According to this model, the electron mean free path in the $\sim 0.3 \mathrm{MV}$ range (100 keV kinetic energy) is slightly rigidity dependent, and its absolute value depends on the characteristics of the dissipation range (spectral index and wavenumber where it sets in) and the resonance broadening. A comparison of the model predictions with the mean free paths obtained by fitting Wind observations of $\sim 25-500 \mathrm{keV}$ electrons with an interplanetary transport model showed good agreement (Dröge 2003, 2005), suggesting a relation between the properties of solar particle transport and solar wind turbulence on an event-by-event basis.

In this study, we obtained a picture for the properties of particle scattering consistent with these previous studies. We found some variation in the magnitudes of the radial mean free path from one event to another, ranging from 0.12 to $0.44 \mathrm{AU}$. These values were obtained from an analysis of the evolution of the PADs, and the values inferred using ACE and Wind observations for the same event were consistent. The results obtained by fitting each energy separately suggest that the electron radial mean free path is rigidity dependent in the range from $0.3 \mathrm{MV}$ to $0.5 \mathrm{MV}$. Figure 5 shows the values of the radial mean free 


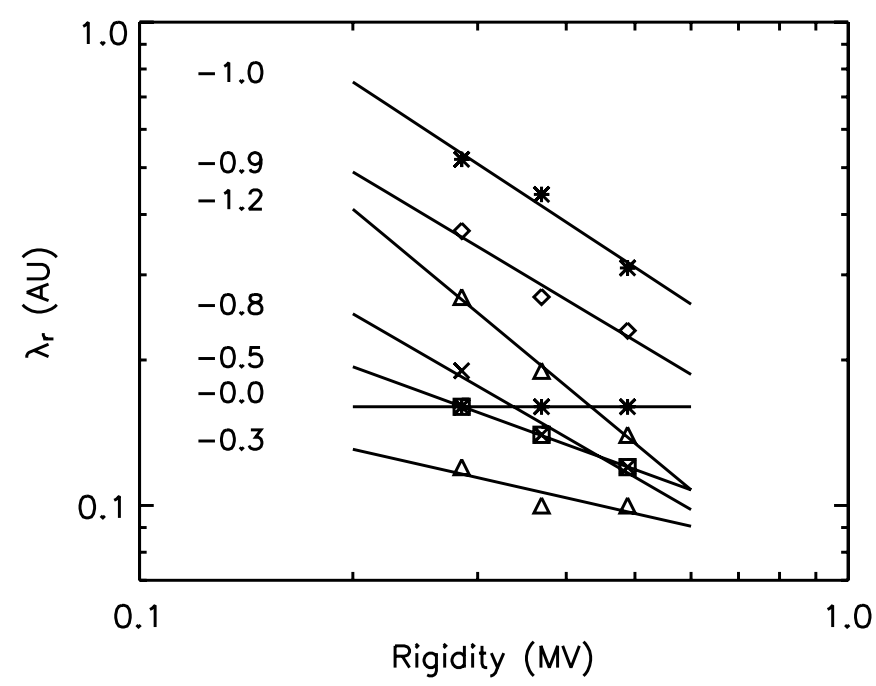

Fig. 5. Radial mean free path vs. particle rigidity for the selected SEP events. Each event is displayed with a different symbol; the values listed on the left are the slopes for each event.

path obtained as a function of rigidity (in each energy channel). The tendency suggests increasing values of $\lambda_{r}$ toward smaller rigidities with a mean spectral slope of -0.7 . Although the values derived for this set of events are not self-consistent (rigidity dependence should be included in the numerical simulations to take it properly into account), they provide further support to the rigidity dependence of the scattering mean free path of NR electrons.

\section{Conclusions}

Electrons observed in interplanetary space appear to be released during either short $(<30 \mathrm{~min})$ or long $(>2 \mathrm{~h})$ periods on a sample of seven well-observed events. Electron events associated with short release episodes appear to originate in solar flares. We find a correspondence between the timing of the release and the observed type III radio bursts. Magnetic connectivity plays an important role in space. Only type III radio bursts reaching the plasma line near the Earth have an associated release episode onto the spacecraft-connected field line.

Electron events consistent with long release episodes are associated with signatures of long acceleration processes in the low corona (long decay SXR emission, type IV radio bursts, and time-extended microwave emission). This kind of emission is only missing for one event in this group, for which, however, a long-lasting type II burst was reported, suggesting that the acceleration of NR electrons might be related to a coronal shock. Since type II radio bursts were reported for many of the selected events $(5 / 7)$, the emission does not seem to be a conclusive signature to pinpoint the release timescales of the particles observed in situ.

The observation of NR electron events showing beamed PADs during several hours is the clearest observational signature of sustained release in the corona. On the other hand, the observation of in situ PADs isotropizing in less than a couple of hours is a clear signature of a prompt release of electrons in the low corona, as proposed by Kahler et al. (2007) in a previous observational study. In this sense, Wind/3DP observations are unique, because 3DP is the only experiment in space able to provide us with measurements of the complete PADs. It is thus imperative to develop new techniques to optimize the scientific return of experiments on board future spacecraft, such as Solar Orbiter and Solar Probe +, that will measure PADs with only a limited number of fields of view.

Acknowledgements. The research leading to these results received funding from the European Union's Seventh Framework Programme (FP7/2007-2013) under grant agreement No. 262773 (SEPServer). N.A. and B.S. were also supported by the Ministerio de Economía y Competitividad (Spain), under the projects PN AYA2010-17286 and AYA2013-42614. N.A. acknowledges support by the Agència de Gestió d'Ajuts Universitaris i de Recerca (AGAUR) to visit the Observatorie de Paris in Meudon during two months. E.T. and R.V. acknowledge the support from the Academy of Finland (project 133723). The SOHO/LASCO CME catalog is generated and maintained at the CDAW Data Center by NASA and The Catholic University of America in cooperation with the Naval Research Laboratory. SOHO is a project of international cooperation between ESA and NASA.

\section{References}

Agueda, N., \& Klein, K.-L. 2013, Proc. IAU Symp. No. 300, 8, 481 Agueda, N., \& Vainio, R. 2013, J. Space Weather Space Clim., 3, A260000 Agueda, N., Vainio, R., Lario, D., \& Sanahuja, B. 2008, ApJ, 675, 1601

Agueda, N., Lario, D., Vainio, R., et al. 2009a, A\&A, 507, 981

Agueda, N., Vainio, R., Lario, D., \& Sanahuja, B. 2009b, Adv. Space Res., 44, 794

Agueda, N., Vainio, R., Lario, D., \& Sanahuja, B. 2010, A\&A, 519, A36

Agueda, N., Vainio, R., \& Sanahuja, B. 2012, ApJS, 202, 18

Aschwanden, M. J. 2002, Space Sci. Rev., 101, 1

Beeck, J., \& Wibberenz, G. 1986, ApJ, 311, 437

Bougeret, J.-L., Kaiser, M. L., Kellogg, P. J., et al. 1995, Space Sci. Rev., 71, 231

Cane, H. V. 2003, ApJ, 598, 1403

Dalla, S., Marsh, M. S., Kelly, J., \& Laitinen, T. 2013, J. Geophys. Res., 118, 5979

Dresing, N., Gómez-Herrero, R., Klassen, A., et al. 2012, Sol. Phys., 281, 281

Dröge, W. 2000, ApJ, 537, 1073

Dröge, W. 2003, ApJ, 589, 1027

Dröge, W. 2005, Adv. Space Res., 35, 532

Dröge, W., Kartavykh, Y. Y., Klecker, B., \& Kovaltsov, G. A. 2010, ApJ, 709, 912

Gold, R. E., Krimigis, S. M., Hawkins, III, S. E., et al. 1998, Space Sci. Rev., 86, 541

Haggerty, D. K., \& Roelof, E. C. 2002, ApJ, 579, 841

Haggerty, D. K., \& Roelof, E. C. 2003, Adv. Space Res., 32, 423

Huttunen-Heikinmaa, K., Valtonen, E., \& Laitinen, T. 2005, A\&A, 442, 673

Jokipii, J. R. 1966, ApJ, 146, 480

Kahler, S., \& Ragot, B. R. 2006, ApJ, 646, 634

Kahler, S. W., Aurass, H., Mann, G., \& Klassen, A. 2007, ApJ, 656, 567

Kartavykh, Y. Y., Dröge, W., \& Klecker, B. 2013, J. Geophys. Res., 118, 4005

Kelly, J., Dalla, S., \& Laitinen, T. 2012, ApJ, 750, 47

Klein, K.-L., Krucker, S., Trottet, G., \& Hoang, S. 2005, A\&A, 431, 1047

Klein, K.-L., Krucker, S., Lointier, G., \& Kerdraon, A. 2008, A\&A, 486, 589

Klein, K.-L., Trottet, G., \& Klassen, A. 2010, Sol. Phys., 263, 185

Kocharov, L., Vainio, R., Kovaltsov, G. A., \& Torsti, J. 1998, Sol. Phys., 182, 195

Krucker, S., Larson, D. E., Lin, R. P., \& Thompson, B. J. 1999, ApJ, 519, 864

Krucker, S., Kontar, E. P., Christe, S., \& Lin, R. P. 2007, ApJ, 663, L109

Laitinen, T., Huttunen-Heikinmaa, K., \& Valtonen, E. 2010, 12th Int. Solar Wind Conf., 1216, 249

Lario, D., Roelof, E. C., Decker, R. B., \& Reisenfeld, D. B. 2003, Adv. Space Res., 32, 579

Li, B., \& Cairns, I. H. 2013, ApJ, 763, L34

Lin, R. P., Anderson, K. A., Ashford, S., et al. 1995, Space Sci. Rev., 71, 125

Lin, R. P., Dennis, B. R., Hurford, G. J., et al. 2002, Sol. Phys., 210, 3

Lintunen, J., \& Vainio, R. 2004, A\&A, 420, 343

Maia, D. J. F., \& Pick, M. 2004, ApJ, 609, 1082

Maia, D. J. F., Gama, R., Mercier, C., et al. 2007, ApJ, 660, 874

Malandraki, O. E., Agueda, N., Papaioannou, A., et al. 2012, Sol. Phys., 281, 333 
A\&A 570, A5 (2014)

Marsh, M. S., Dalla, S., Kelly, J., \& Laitinen, T. 2013, ApJ, 774, 4 Meyer-Vernet, N., \& Perche, C. 1989, J. Geophys. Res., 94, 2405

Poquérusse, M. 1994, A\&A, 286, 611

Roelof, E. C. 1969, in Lectures in High-Energy Astrophysics, eds. H. Ögelman, \& J. R. Wayland (The Scientific and Technical Information Division Office of Technology Utilization Naza), 111

Rouillard, A. P., Sheeley, N. R., Tylka, A., et al. 2012, ApJ, 752, 44

Ruffolo, D. 1995, ApJ, 442, 861

Ruffolo, D., Khumlumlert, T., \& Youngdee, W. 1998, J. Geophys. Res., 103, 20591

Sáiz, A., Evenson, P., Ruffolo, D., \& Bieber, J. W. 2005, ApJ, 626, 1131
Simnett, G. M., Roelof, E. C., \& Haggerty, D. K. 2002, ApJ, 579, 854

Stone, R. G., Bougeret, J. L., Caldwell, J., et al. 1992, A\&AS, 92, 291

Tan, L. C., Reames, D. V., Ng, C. K., Shao, X., \& Wang, L. 2011, ApJ, 728, 133

Vainio, R., Valtonen, E., Heber, B., et al. 2013, J. Space Weather Space Clim., 3, A260000

Wibberenz, G., \& Cane, H. V. 2006, ApJ, 650, 1199

Wiedenbeck, M. E., Mason, G. M., Cohen, C. M. S., et al. 2013, ApJ, 762, 54

Yashiro, S., Gopalswamy, N., Michalek, G., et al. 2004, J. Geophys. Res., 109, 7105 